\title{
Numerical Investigation of Acoustics Performance of Low- Pressure Ducted Axial Fan by Using Different Turbulence Models
}

\author{
Aykut Bacak ${ }^{*}$ and Ali Pinarbasi \\ Yıldız Technical University, Mechanical Engineering Department, 34349 Besiktas, Istanbul, Turkey \\ *Corresponding Author: bacakaykut@gmail.com
}

\begin{abstract}
In this article, capacity and acoustics parameters of low pressure ducted axial fan is numerically investigated with Realizable kepsilon, k-w SST and DES turbulence models by using computational fluid dynamics software. One slice of six bladed axial fan operating at 3000 RPM is simulated periodically as low pressure ducted axial ventilation fan. Simulations are run for operating point on the performance curve for each turbulence models. Investigation of acoustics parameters are obtained Ffowcs-Williams Hawkings acoustic model to calculate sound pressure levels for related frequencies. Numerical results are compared with the experimental results provided from blade manufacturer company.
\end{abstract}

\section{Introduction}

Axial flow fans are the continuous flow machines that air or any other gas flows through in an approximately axial direction by using rotational blades inside them. Axial fans are generally used at buildings, industrial areas cars and public transport vehicles ventilating and/or air conditioning equipment [1]. The term of ducted fan is defined by the sum of air or other gas which it forces through the system against resistance representing skin friction, pressure loses and secondary flows. Ducted axial fans contain blades with aerodynamic cross-section, electric motor, construction support elements and other auxiliary equipment [2]. Axial fans generate high or low noise levels while operating at high or low rotational speed for different reasons. The increase in the use of high-rise buildings in big cities, and therefore the increased use of axial fan, will lead to noise pollution. Therefore, while designing the axial fan blades, it is necessary to examine the acoustic performance while examining parameters such as flow rate, pressure and efficiency.

Sound is always related with fast small-scale pressure fluctuations exceeding the normal atmospheric pressure. These fluctuations are radiated from sources and travel as waves through the medium at sound speed. Sound may be reflected, particularly absorbed 
or reduced before getting at the human eardrum where it generates a sensation of hearing rely on the amplitude of the sound wave [3]. Neise [4], defined aeroacoustics sound generation mechanism for fans like shown in Figure 1. According that, the most common noise mechanism occurs from the unsteady-rotating forces some of them can be regards as performance loses such as secondary flows and vortex shedding.

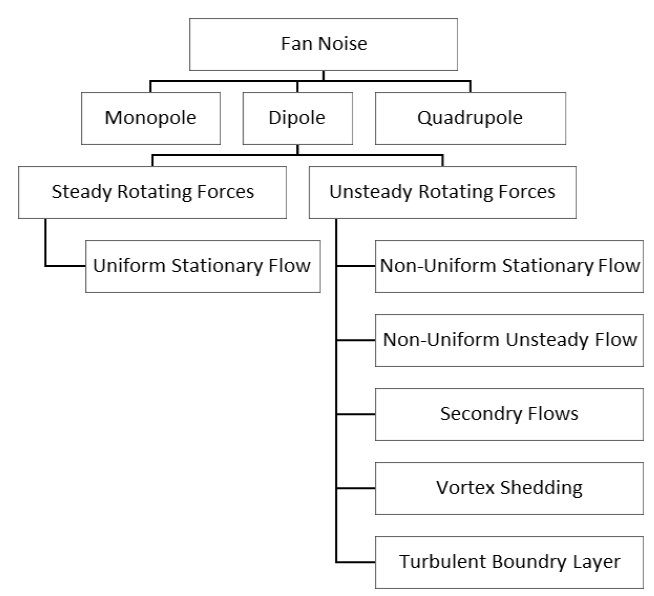

Fig. 1. Overview of the aeroacoustics sound generation mechanisms for fans.

Noise generated by axial fan can be reduced by optimizing electrical motor noise and aerodynamically generated noise. Any kind of blade design with different size and shape can be investigated by using numerical methods with different case studies to research noise generating parameters such as sound pressure and power levels to optimize aero-acoustically. There are many great efforts both in industrial area and scientific society to reduce ventilation fan noise levels by using different type of computational fluid dynamics software and numerical methods in literature. Sturm et al. [5], investigated tonal sound pressures of axial fan rotor by using Lattice Boltzmann approach for geometrically different test cases. They obtained relatively close SPL results for all cases and similar results to experimental ones. Reese at al. [6], investigated the acoustics performance of an axial fan by using different turbulence models such as URANS, DES, SAS and LES. They obtained sound pressure power spectral density results by using virtual sound receptor located at one meter far-away from the fan with $45^{\circ}$ angle from the axis. They obtained the closest results to experimental studies at LES turbulence model. Perot et al. [7], investigated an axial fan aerodynamic and aeroacoustics performance numerically and experimentally with Lattice-Boltzmann method. They consider the influence of inlet flow on BPF noise in their studies. They stated importance of modeling the geometrical details of a hood to capture the BPF. They attained a great agreement for numerical and experimental results. Marsan et al. [8], investigated the tip leakage and its impact of lowspeed fan numerically with Lattice-Boltzmann Method as three different voxel sizes and compared numerical sound pressure level of same fan with reference experimental results. 


\section{Numerical Method}

In this paper, all simulations are made by using Ansys 18.1 Fluent, computational fluid dynamics software. Simulating process consists of design, meshing, fluid flow modeling and solution process. Basis steps of simulation process such as defining properties of simulation, applying boundary conditions and selecting appropriate turbulence model are applied at flow modeling section. In this paper, one slice of six bladed axial fan operating at 3000 RPM is simulated periodically with Realizable k- $\varepsilon, k-w$ SST and DES turbulence models at operating point.

Dimensions of domain is taken from ANSI/ AMCA 320-08 [9]. Upstream and downstream length is taken $10 \mathrm{D}$ and the radius of expansion domain is taken $3 \mathrm{D}$ where $\mathrm{D}$ is the hydraulic diameter of axial fan. Fluid domain consists of velocity inlet, pressure outlet, periodically rotating hub and blade walls, stationary and periodic walls as boundary conditions. Rotating blade and hub walls with shroud surface defined as noise sources like shown in Figure 1 in a large scale. Around 800,000 triangular mesh elements and 10 inflation layers with $0,008 \mathrm{~mm}$ first layer thickness near the wall are used for all simulations. Static pressure measurement is done just after and before from rotating domain and velocity measurement is taken from $8.5 \mathrm{D}$ faraway from fan in the downstream flow. As it is defined at ANSI/AMCA 210-07 Standard [10], velocity measurements are taken at defined location with identified measurement points. Mass-weighted Average method at that location is used for velocity measurement instead of measuring identified measurement points. Static pressure drop is also measured with Mass-weighted average method since hub prevents the fluid flow around the axis.

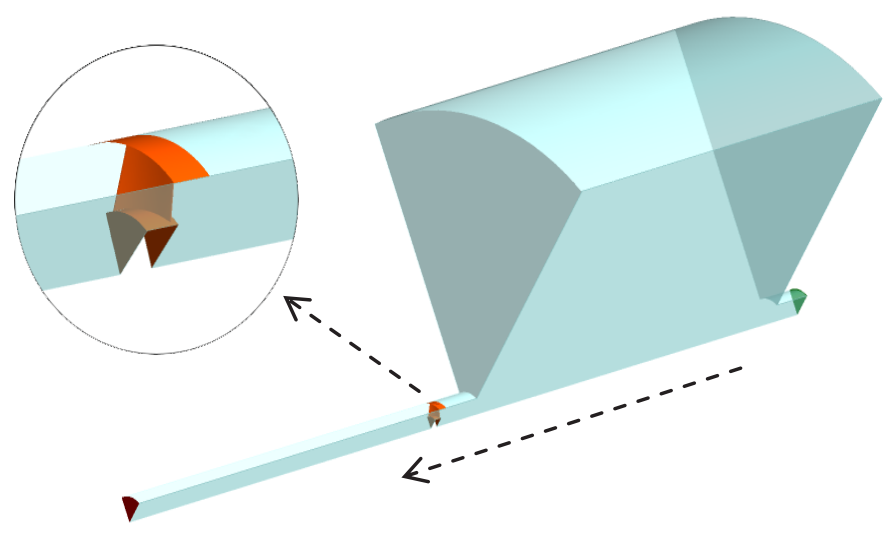

Fig. 2. Fluid domain of numerical simulation.

Ffows-William Hawkings acoustic module is used to obtain acoustic parameters with 2E-05 Pa reference acoustic pressure, $340 \mathrm{~m} / \mathrm{s}$ sound speed and $1.225 \mathrm{~kg} / \mathrm{m}^{3}$ air density as boundary conditions. Virtual noise measurement sensors are placed $2 \mathrm{~m}$ far-away with $45^{\circ}$ angle from the axis of the fan upstream in the expansion domain not to be influenced from the fluid flow like shown in Figure 3 as experimental measurement. 


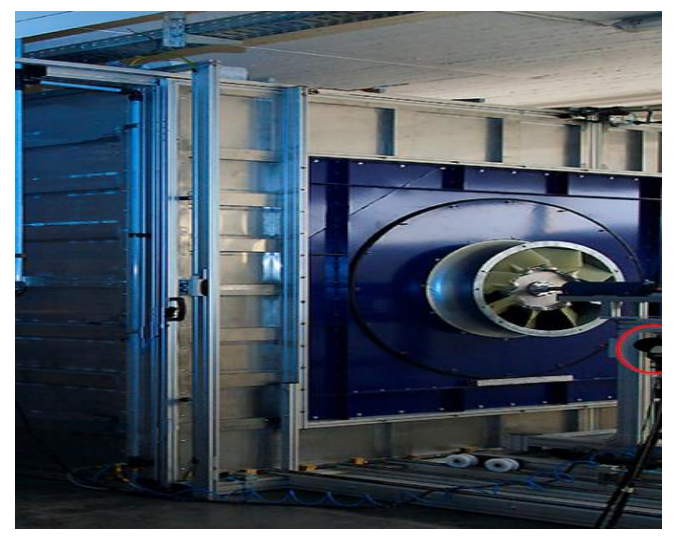

Fig. 3. Experimental acoustic performance measurement provided by manufacturer.

Continuity and Navier-Stokes Equations coupled with k- $\varepsilon, k-\omega$ SST and DES turbulence models are used in fan simulations. Continuity, momentum and k- $\varepsilon, k-\omega$ SST and DES turbulence model equations are given below;

The Mass Conservation Equation;

$$
\frac{\partial \rho}{\partial t}+\nabla(\rho \vec{v})=0
$$

where $\mathrm{v}$ is velocity and $\rho$ is the density [11].

Momentum Conservation Equation;

$$
\begin{gathered}
\frac{\partial(\rho \vec{v})}{\partial t}+\nabla(\rho \vec{v} \vec{v})=\nabla P+\nabla \cdot(\bar{\tau})+\rho \vec{g}+\vec{F} \\
\bar{\tau}=\mu\left[\left(\nabla \vec{v}+\nabla \vec{v}^{T}\right)-\frac{2}{3} \nabla \vec{v} I\right]
\end{gathered}
$$

where $\mathrm{P}$ is the static pressure, $\bar{\tau}$ is the stress tensor, $\rho \vec{g}$ and $\vec{F}$ are the gravitational body force and external body forces respectively, $\mu$ is the molecular viscosity and $I$ is the unit tensor. [11].

Transport equations for realizable k- $\varepsilon$ turbulence model;

$$
\begin{gathered}
\frac{\partial(\rho k)}{\partial t}+\frac{\partial\left(\rho k u_{j}\right)}{\partial x_{j}}=\frac{\partial}{\partial x_{j}}\left[\left(\mu+\frac{\mu_{t}}{\sigma_{k}}\right) \frac{\partial k}{\partial x_{j}}\right]+G_{k}+G_{b}-\rho \varepsilon-Y_{M}+S_{k} \\
\frac{\partial(\rho \varepsilon)}{\partial t}+\frac{\partial\left(\rho \varepsilon u_{j}\right)}{\partial x_{j}}=\frac{\partial}{\partial x_{j}}\left[\left(\mu+\frac{\mu_{t}}{\sigma_{\varepsilon}}\right) \frac{\partial \varepsilon}{\partial x_{j}}\right]+\rho C_{1} S \varepsilon-\rho C_{2} \frac{\varepsilon^{2}}{k+\sqrt{v \varepsilon}}+C_{1} \varepsilon \frac{\varepsilon}{k} C_{3 \varepsilon} G_{b}+S_{\varepsilon}
\end{gathered}
$$




$$
\begin{gathered}
C_{1}=\max \left[0.43, \frac{\eta}{\eta+5}\right] \\
\eta=S \frac{k}{\varepsilon} \\
S=\sqrt{2 S_{i j} S_{i j}}
\end{gathered}
$$

Where $G_{k}$ represents the generation of turbulence kinetic energy due to mean velocity gradients, $G_{b}$, is the generation of turbulence kinetic energy due to buoyancy, $Y_{M}$, represents the contribution of the fluctuating dilatation in compressible turbulence to the overall dissipation rate, $\mathrm{C}_{2}$ and $\mathrm{C}_{1 \varepsilon}$ are constants, $\sigma_{\mathrm{k}}$ and $\sigma_{\varepsilon}$ are the turbulence Prandtl numbers for $\mathrm{k}$ and $\varepsilon$, respectively. $\mathrm{S}_{\mathrm{k}}$ and $\mathrm{S}_{\varepsilon}$ are user-defined source terms [11].

Transport equations for $\mathrm{k}-\omega \mathrm{SST}$ turbulence model;

$$
\begin{aligned}
& \frac{\partial(\rho k)}{\partial t}+\frac{\partial\left(\rho k u_{i}\right)}{\partial x_{i}}=\frac{\partial}{\partial x_{j}}\left(\Gamma_{k} \frac{\partial k}{\partial x_{j}}\right)+G_{k}-Y_{k}+S_{k} \\
& \frac{\partial(\rho \omega)}{\partial t}+\frac{\partial\left(\rho \omega u_{j}\right)}{\partial x_{j}}=\frac{\partial}{\partial x_{j}}\left(\Gamma_{\omega} \frac{\partial \omega}{\partial x_{j}}\right)+G_{\omega}-Y_{\omega}+S_{\omega}
\end{aligned}
$$

where $G_{k}$ represents the generation of turbulence kinetic energy due to mean velocity gradients, $G_{\omega}$, represents the generation of $\omega, \Gamma_{\mathrm{k}}$ and $\Gamma_{\omega}$ represent the effective diffusivity of $\mathrm{k}$ and $\omega$, respectively, $\mathrm{Y}_{\mathrm{k}}$ and $\mathrm{Y}_{\omega}$ represent the dissipation of $\mathrm{k}$ and $\omega$ due to turbulence, $\mathrm{S}_{\mathrm{k}}$ and $\mathrm{S}_{\omega}$ are user-defined source terms [11].

DES with the SST k- $\omega$ model equations;

$$
\begin{gathered}
Y_{k}=\rho \beta^{*} k \omega F_{D E S} \\
F_{D E S}=\max \left(\frac{L_{t}}{C_{D E S} \Delta_{\max }}, 1\right) \\
L_{t}=\frac{\sqrt{k}}{\beta^{*} \omega} \\
F_{D E S}=\max \left(\frac{L_{t}}{C_{D E S} \Delta_{\max }}\left(1-F_{S S T}, 1\right)\right.
\end{gathered}
$$

where $\Delta_{\max }$ is the maximum local grid spacing $\left(\Delta_{x}, \Delta_{y}, \Delta_{z}\right), C_{D E S}$ is a calibration constant used in DES model has a value of 0.61 [11].

$\frac{1}{a_{0}^{2}} \frac{\partial^{2} p^{\prime}}{\partial t^{2}}-\nabla^{2} p^{\prime}=\frac{\partial^{2}\left(T_{i j} H(f)\right)}{\partial x_{i} \partial x_{j}}-\frac{\partial}{\partial x_{j}}\left(\left[P_{i j} n_{j}+\rho u_{i}\left(u_{n}-u_{v}\right)\right] \delta(f)\right)+\frac{\partial}{\partial t}\left(\left[\rho_{0} v_{n}+\rho\left(u_{n}-u_{v}\right)\right] \delta(f)\right)$ 
where $u_{i}$ is fluid velocity component in the $x_{i}$ direction, $u_{n}$ is fluid velocity component normal to the surface $\mathrm{f}=0, \mathrm{v}_{\mathrm{i}}$ is surface velocity components in the $\mathrm{x}_{\mathrm{i}}$ direction, $\mathrm{v}_{\mathrm{n}}$ is surface velocity component normal to the surface, $\delta(f)$ is the dirac delta function and $H(f)$ is Heaviside function.

\section{Result and Discussion}

In this study an axial fan operating at around $6800 \mathrm{~m}^{3} / \mathrm{h}$ and $285 \mathrm{~Pa}$ (Static) is numerically investigated to obtain sound pressure levels between 63 and $8000 \mathrm{~Hz}$ by using k-epsilon, k-w SST and DES turbulence models. It has been seen that pressure drop values measured just after and before from the fan rotor and axial velocity values calculated as mass average weighted from indicated location described previously are in acceptable levels for all cases. Sound pressure levels, shown in Figure 3, obtained by virtual receivers located $2 \mathrm{~m}$ far away from the fan rotor and numerical simulations run for around 100 seconds. Sound pressure levels at different octave band are obtained from Fast Fourier Transform (FFT) in same CFD Software. As it can be seen from Figure 3, total sound pressure level values are in acceptable values with provided values from manufacturers.

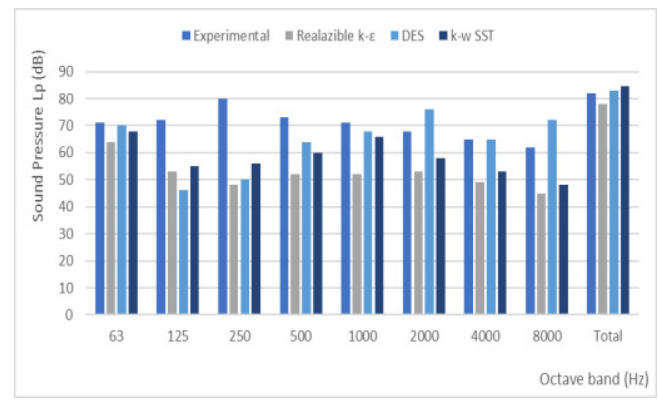

Fig. 4. Sound pressure levels results at octave band.

Dipole source strength, turbulence kinetic energy and turbulence eddy dissipation contours obtained by realizable $\mathrm{k}$-epsilon turbulence model at $\mathrm{z}=0$ location (center plane) are shown in Figure 4. It is useful to investigate dipole source strength contour at center plane, since most of noise generated by axial fan is dipole as it is described first chapter as table. At it is examined turbulence kinetic energy contour, it is seen that kinetic energy distribution is similar the dipole source strength distribution. So, reducing the kinetic energy around fan blade may reduce the noise levels. Turbulence eddy dissipation contour is also similar the dipole source strength distribution. Reducing eddy dissipation around fan blade may be another solution for reducing the noise levels of fans.

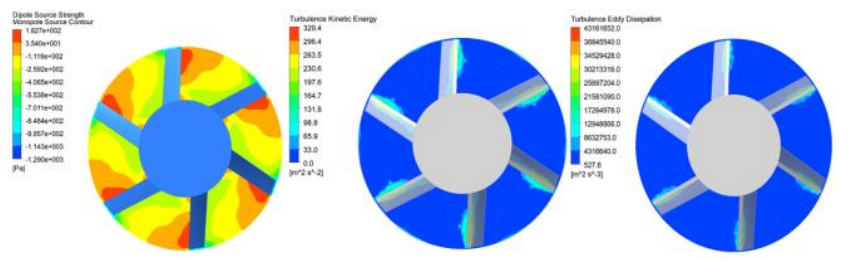

Fig. 5. Dipole source strength, turbulence kinetic energy and turbulence eddy dissipation contour showing at the same plane 


\section{Conclusion}

In this study, some numerical investigations on the airflow and acoustics performances of an axial ventilation fan are performed. The analysis is based on the aeroacoustic analogy where the CFD computations have been performed using Ansys Fluent 18.1. Numerical sound pressure level values obtained by CFD calculations are with values provided by axial fan blade manufacturer. Acceptable agreement has been observed between numerical and provided sound pressure levels. Good agreement has also been observed for pressure drop and flow rate provided values.

\section{REFERENCES}

[1] F. P. Bleier, Fan Handbook Selection, Application, and Design, The USA: McGrawHill, (1997).

[2] R. A. Wallis, Axial Flow Fans Design and Practice, New York and London: Academic Press, (1961).

[3] S. Wagner, R. Bareib, G. Guidati, Wind Turbin Noise, Germany: Springer, (1996).

[4] W. Neise, "Review of Fan Noise Generation Mechanicsm and Control Methods," in Fan Noise Symposium CETIM, France , (1992).

[5] Sturm M., Sanjose M., Moreau S., Carolus T., "Aeroacoustic Simulation of an Axial Fan Including the Full Test Rig by Using Lattice Boltzmann Method," in Fan 2015 Conference, (2015).

[6] Reese H., Carolus T., Kato C., "Numerical Prediction of the Aeroacoustic Sound Sources in a Low-Pressure Axial Fan with Inflow Distortion," in Fan Conference 2007, Lyon, France, (2007).

[7] Perot F., Kim M. S., Moreau S., Henner M., "Axial Fan Noise Aeroacoustics Predictions and Inflow Effect on Tonal Noise using LBM," in CFD Canada 2013 Conference, Canada, (2013).

[8] Marsan A., Daniels D. L., Sanose M., Moreaus S., "Tip Leakage Flow and its Implication on the Acoustic Signature of a Low-Speed Fan," in Fan 2018 Conference, Germany, (2018).

[9] ANSI/AMCA 320-08 Laboratory Methods of Sound Testing of Fans Using Sound Intensity, (2013).

[10] ANSI/AMCA 210-07 Laboratory Methdos of Testing Fans for Certified Aerodynamic Performance Rating, (2007).

[11] ANSYS Fluent Thery Guide, ANSYS, Inc., (2013). 